\title{
External Ear Pain
}

National Cancer Institute

\section{Source}

National Cancer Institute. External Ear Pain. NCI Thesaurus. Code C78285.

Painful sensation in the external ear region. 